\title{
DIFFEOMORPHISMS WITHOUT PERIODIC POINTS
}

\author{
J. F. PLANTE
}

\begin{abstract}
It is proved that a compact smooth manifold admits a selfdiffeomorphism without periodic points if and only if its Euler characteristic is zero. When the manifold has dimension $\neq 3$ it is shown that such a diffeomorphism exists which is also volume preserving. The proof of this latter result uses a result of Gromov concerning the existence of nonsingular divergence-free vector fields, so an alternate proof of (jromov's result is sketched.
\end{abstract}

Introduction. Let $M$ be a compact $C^{\infty}$ manifold and $f: M \rightarrow M$ a homeomorphism. A point $p$ in $M$ is a periodic point for $f$ if for some positive integer $n, f^{n}(p)=p$. A homeomorphism corresponds to an action of the integers on $M$ given by $(m, x) \mapsto$ $f^{m}(\dot{x})$. In this note, we are interested in determining whether such an action can be free, which is equivalent to the nonexistence of periodic points for $f$.

TheOREM A. Let $M$ be a compact $C^{\infty}$ manifold. Then $M$ admits a homeomorphism (or diffeomorphism) without periodic points if and only if, $\chi(M)=0$.

TheOREM B. Let $M$ be a compact oriented $C^{x}$ manifold of dimension $\neq 3$. Then $M$ admits a volume preserving diffeomorphism without periodic points if and only if $\chi(M)=0$.

The methods of proof given below give no indication of whether analogous results are true for topological manifolds. Note that in the statement of Theorem B it does not matter whether the volume to be preserved is selected in advance since according to Moser [3], any two volume forms are equivalent via a diffeomorphism. The dimension restriction in Theorem $\mathrm{B}$ is for the benefit of the proof we give, and may not be necessary. In the course of proving Theorem $B$, we will use the following result of Gromov [2] concerning the existence of nonsingular divergence-free vector fields.

THEOREM C. Let $X$ be a nowhere-vanishing vector field on a compact oriented (with volume element) manifold of dimension $\geqslant 3$. Then $X$ is homotopic, through nowherevanishing vector fields, to a divergence-free vector field.

Proof of Theorem C. Since [2] is not too well known, we will sketch a proof which deduces it from the better-known result of Thurston [6] that every transversely oriented codimension one plane field is homotopic to an integrable one. Thus, we

Received by the editors September 13, 1982.

1980 Mathematics Subject Classification. Primary 57R50, 58F20.

Key words and phrases. Diffeomorphism, volume preserving, periodic point, vector field, divergence-free, homotopy. 
may assume that the vector field $X$ is transverse to codimension one foliation. We claim that the plane field $F$ tangent to the foliation can be perturbed to a plane field with adequate "transverse circulation." If the foliation has no compact leaves, a well-known argument shows that through any given point in $M$ there passes an embedded circle which is everywhere transverse to $F$. When the foliation has compact leaves (specifically, bounding dead-end components) it is necessary to perturb $F$ to achieve this situation. This can be done locally. Let $\mathbf{R}^{n}=\mathbf{R}^{3} \times \mathbf{R}^{n-3}$ (since $n \geqslant 3$ ) and give $\mathbf{R}^{3}$ cylindrical coordinates $(r, \theta, z)$. Suppose $F$ is the plane field tangent to the foliation with leaves $z=$ const. Let $\lambda, \mu: \mathbf{R} \rightarrow \mathbf{R}$ be $C^{\infty}$ bump functions such that $\lambda \equiv 0$ outside $(0,1), \mu \equiv 0$ outside $(-2,2)$ and $\mu \equiv 1$ on $[-1,1]$. Let $F^{\prime}=\operatorname{ker} \omega$ where $\omega$ is the one form $d z+\lambda(r) \mu(z) d \theta$. $\omega$ is not integrable; indeed, the planes of $F^{\prime}$ are arranged like the blades of a fan. It is easy to construct embedded circles transverse to $F^{\prime}$. (Descend by a helix with small slope at $r=\frac{1}{2}$ and return vertically.) Choose a finite number of disjoint foliation boxes and perturbations as above such that every compact leaf of the foliation meets the region $\mu \equiv 1$ of at least one of the foliation boxes. At this stage, we can assume that $X$ is transverse to a codimension one plane field $F$ such that every point of $M$ lies on an embedded circle transverse to the plane field. Let $\eta$ be a globally defined one form such that $F=\operatorname{ker} \eta$ and $\eta(X)>0$. Given a point $p$ in $M$, we construct a vector field $X_{p}$ on $M$ such that $X_{p}$ is divergence-free, $\eta\left(X_{p}\right)>0$ whenever $X_{p} \neq 0$, and $X_{p}(p) \neq 0$. (Take an embedded circle through $p$. Construct $X_{p}$ to have the circle as a periodic orbit and have support in a tubular neighborhood of the circie.) Select points $p_{1}, \ldots, p_{k}$ such that the sum of the vector fields $X_{i}=X_{p_{i}}$ is nonvanishing. Let $X^{*}=\sum_{i=1}^{k} X_{i}$, $X_{t}=(1-t) X+t X^{*} . X_{t}$ is nowhere-vanishing since $\eta\left(X_{t}\right)$ is, $X_{0}=X$, and $X_{1}=X^{*}$. Since $X^{*}$ is divergence-free, Theorem $\mathrm{C}$ is proved.

It is worth noting that Theorem $\mathrm{C}$ is true in dimension 1 but false in dimension 2.

Proof of Theorem A. The fact that $\chi(M) \neq 0$ implies the existence of a periodic point is due to Fuller [1]. When $\chi(M)=0$, there exists a nowhere vanishing vector field which may be perturbed by the Kupka-Smale theorem [4] to a nowhere-vanishing vector field whose flow has only hyperbolic periodic orbits. In particular, the set $P$ of all rational multiples of periods of periodic orbits will be a countable set. If $\phi_{t}$ denotes the flow of the vector field and $\tau \notin P$ then the diffeomorphism $f=\phi_{\tau}$ has no periodic points.

Proof of Theorem B. The "only if" part is again Fuller's result. When $M$ has dimension $\geqslant 4$ the Kupka-Smale theorem is valid for divergence-free vector fields according to Robinson [5]. Arguing as in the proof of Theorem A, Theorem B follows for dimension $\geqslant 4$. When $M$ has dimension 1 or 2 ( $M$ is the circle or the torus since it is orientable and $\chi(M)=0), M$ admits a locally free circle action. By averaging, we may assume that the action preserves a smooth volume element. Regarding the action as a flow, we again determine that the set of rational multiples of periods of the flow is countable and argue as before. This completes the proof of Theorem B.

REMARK. The argument just used in dimension $\leqslant 2$ also works for Seifert 3-manifolds. 


\section{REFERENCES}

1. F. Fuller, The existence of periodic points, Ann. of Math. (2) 57 (1953), 229-230.

2. M. Gromov, Convex integration of differential relations, Izv. Akad. Nauk 37 (1973), 329-343. (Russian)

3. J. Moser, On the volume elements on a manifold. Trans. Amer. Math. Soc. 120 (1965), 286-294.

4. M. Peixoto, On an approximation theorem of Kupka and Smale, J. Differential Equations 3 (1967). 214-227.

5. R. Robinson, Generic properties of conservative systems, Amer. J. Math. 92 (1970), 562-603.

6. W. Thurston. Existence of codimension one foliations, Ann. of Math. (2) 104 (1976), 249-268.

Department of Mathematics, University of North Carolina at Chapel Hill. Chapel Hill, NORTH CAROLINA 27514 